ARTICLE

https://doi.org/10.1038/s41467-019-12002-1

\title{
Auxin-sensitive Aux/IAA proteins mediate drought tolerance in Arabidopsis by regulating glucosinolate levels
}

Mohammad Salehin ${ }^{1}$, Baohua Li ${ }^{2}$, Michelle Tang ${ }^{2}$, Ella Katz ${ }^{2}$, Liang Song ${ }^{3}$, Joseph R. Ecker (i) ${ }^{3}$, Daniel J. Kliebenstein (D $^{2} \&$ Mark Estelle ${ }^{1}$

A detailed understanding of abiotic stress tolerance in plants is essential to provide food security in the face of increasingly harsh climatic conditions. Glucosinolates (GLSs) are secondary metabolites found in the Brassicaceae that protect plants from herbivory and pathogen attack. Here we report that in Arabidopsis, aliphatic GLS levels are regulated by the auxin-sensitive Aux/IAA repressors IAA5, IAA6, and IAA19. These proteins act in a transcriptional cascade that maintains expression of GLS levels when plants are exposed to drought conditions. Loss of IAA5/6/19 results in reduced GLS levels and decreased drought tolerance. Further, we show that this phenotype is associated with a defect in stomatal regulation. Application of GLS to the iaa5,6,19 mutants restores stomatal regulation and normal drought tolerance. GLS action is dependent on the receptor kinase GHR1, suggesting that GLS may signal via reactive oxygen species. These results provide a novel connection between auxin signaling, GLS levels and drought response.

\footnotetext{
${ }^{1}$ Section of Cell and Developmental Biology and Howard Hughes Medical Institute, University of California, San Diego, La Jolla CA. 92093, USA. ${ }^{2}$ Department of Plant Sciences, University of California, Davis, CA 95616, USA. ${ }^{3}$ Genomic Analysis Laboratory, Howard Hughes Medical Institute and The Salk Institute for Biological Studies, La Jolla, CA 92037, USA. Correspondence and requests for materials should be addressed to M.E. (email: mestelle@ucsd.edu)
} 
T he Aux/IAA proteins are a large family of auxin coreceptors and transcriptional repressors that have a central role in auxin signaling. In the presence of auxin, the Aux/ IAAs are degraded through the action of the ubiquitin E3-ligase SCFTIR1/AFB, resulting in de-repression of transcription by the AUXIN RESPONSE FACTOR (ARF) transcription factors ${ }^{1,2}$. Although the mechanisms of auxin perception and Aux/IAA degradation are well known, other aspects of Aux/IAA regulation remain poorly understood. In particular, the factors that regulate transcription of the $A u x / I A A$ genes are mostly unknown. Transcription of many $A u x / I A A s$ genes is auxin regulated via the ARF proteins resulting in robust negative feedback regulation of auxin response. In addition, expression of SHY2/IAA3 by the ARR1 transcription factor is important for cell differentiation in the $\operatorname{root}^{3}$. However, because expression of the $29 A u x / I A A$ genes in Arabidopsis is highly dynamic with both shared and specific expression domains, they are probably regulated by many additional transcription factors. In this way, auxin response pathways can be integrated with other signaling pathways that respond to environmental or genetic signals. As proof of this concept, we recently showed that three $A u x / I A A$ genes, IAA5, IAA6, and IAA19 are directly regulated by DREB2A and DREB2B, transcription factors that are known be important for drought response $^{4}$. Further we demonstrated that these $A u x / I A A$ genes are required for drought tolerance. Here we show that these Aux/ IAAs act to regulate the levels of aliphatic glucosinolates (GLS), well characterized secondary metabolites that contribute to herbivore tolerance and innate immunity. We also demonstrate that GLS compounds have an important role in stomatal regulation and drought tolerance.

\section{Results}

IAA5/6/19 regulate glucosinolate biosynthesis. To determine the molecular basis of reduced drought tolerance in iaa5, iaa6, and iaa19 mutant plants, we used RNAseq to identify genes that were differentially regulated in Col-0 vs. the iaa5 iaa6 iaa19 $($ iaa5,6,19) triple mutant when exposed to desiccation stress (Supplementary Data 1). A total of 651 genes were differentially expressed between the mutant and Col-0 under these conditions $(\mathrm{FDR}<0.001)$, 321 down-regulated and 330 up-regulated. A gene ontology search revealed that 14 genes that function in the aliphatic glucosinolate (GLS) biosynthetic pathway are downregulated in iaa5,6,19 (Fig. 1a, b). In contrast, expression of these genes is not significantly affected by dehydration stress in Col-0. We confirmed some of these results by quantitative RTPCR (qRT-PCR) (Supplementary Fig. 1a).

Because the aliphatic GLS biosynthetic enzymes are downregulated in iaa5,6,19 mutants during drought stress, we wondered if the levels of GLSs were also affected. To test this, we measured GLS levels in stress treated Col-0 and iaa5,6,19 mutants at time intervals. Indolic GLSs were unaltered (Supplementary Table 1). However, the level of 4-methylsulfinyl glucosinolate (4-MSO), the most abundant aliphatic glucosinolate in Arabidopsis (Col-0), was sharply decreased in iaa5, 6, 19 plants after $1 \mathrm{~h}$ and $3 \mathrm{~h}$ of desiccation (Fig. 1c). These data shows that down-regulation of aliphatic GLS biosynthetic enzymes in iaa5, 6, 19 mutants results in decreased GLS levels.

Aliphatic GLSs are required for drought tolerance. GLSs are well known for their role in plant defense and innate immunity, although recent studies also suggest that they may have a role in regulating plant growth ${ }^{5-9}$. These secondary metabolites are found primarily in the Brassicaceae, a family that includes many economically important crops ${ }^{7}$. GLSs are broken down by the enzyme myrosinase into thiohydroximate-O-sulfonates which rearrange to form diverse isothiocyanates (ITC), nitriles and related compounds ${ }^{8-12}$. To determine if decreased drought tolerance in the iaa5,6,19 mutant is related to reduced GLS levels, we measured the effects of mutations in GLS biosynthetic genes on response to drought. We employed two assays; growth of seedlings on agar medium containing PEG, and growth of plants in pots after withholding water. We first determined the response of the iaa5,6,19 mutant to water withholding. The results in Fig. 2a, b) confirm that the mutant is less tolerant to drought conditions. Next we characterized mutants in the CYP79F1 and CYP79F2 genes, encoding enzymes that convert elongated methionine to aldoximes (Fig. 1a). We tested the cyp79f1f2 double mutant and found that it was less tolerant to both PEG treatment and water withholding (Fig. 2c, d; Supplementary Fig. 2a). Similarly, loss of CYP83A1, responsible for conversion of aldoximes to aci-Nitro compounds, results in reduced tolerance to water withholding in pots (Supplementary Fig. 2b). These results indicate that loss of aliphatic GLS compounds results in decreased drought tolerance, providing an explanation for the phenotype of the iaa5,6,19 mutant.

The MYB28 and MYB29 transcription factors are known to regulate genes in the aliphatic GLS biosynthetic pathway, including the CYP79F1/F2 and CYP83A1 genes ${ }^{13-16}$. Indeed, aliphatic GLS levels in the $m y b 28 m y b 29$ double mutant are extremely low, whereas overexpression of either MYB28 or MYB29 results in elevated aliphatic GLS levels ${ }^{14-17}$. Thus, it is possible that the effects of the iaa5,6,19 mutations on the pathway are mediated by changes in expression of these transcription factor genes. Examination of our RNAseq data revealed that MYB28 is down-regulated in the triple mutant in response to stress. MYB29 is expressed at a very low level in seedlings. We confirmed this by qRT-PCR (Supplementary Fig. 1). When we determined the response of the myb28 mby29 double mutant to water withholding we found that it is less tolerant than the wild type, further support for the idea that GLSs are required for drought tolerance (Fig. 2e; Supplementary Fig. 3a). Since overexpression of MYB28 and MYB29 increases GLS levels, we also tested the behavior of these lines in our assays. Strikingly, we found that both lines displayed strongly increased drought tolerance (Fig. 2f, Supplementary Fig. 3b) further confirming that GLSs confer drought tolerance.

Although the role of MYB28/29 in the regulation of GLS biosynthesis is well known, it is possibly that these transcription factors regulate other genes that contribute to drought tolerance. To address this possibility, we also tested the effect of overexpression of the AOP2 gene on drought tolerance. AOP2 converts 4-MSO to but-3-enyl glucosinolate. Arabidopsis Col-0 lacks AOP2 but overexpression of Brassica oleracea AOP2 in Col0 results in an increase in aliphatic GLS levels, albeit less than in lines over-expressing $M Y B 28^{18}$. The results in Fig. $2 \mathrm{f}$ and Supplementary Fig. 3b show that increased AOP2 levels results in a modest but statistically significant increase in drought tolerance confirming that MYB28/29 overexpression affects drought tolerance by increasing GLS levels.

If decreased drought tolerance in iaa5,6,19 plants is due to a GLS deficiency then overexpression of MYB28 or MYB29 in the triple mutant may ameliorate the effects of the mutations. Indeed, when we cross the 35 S:MYB28 or 35 S:MYB29 transgene into the iaa19-1 mutant the result is the restoration of wild-type levels of drought tolerance to the mutant line (Fig. $2 \mathrm{~g}$ ).

WRKY63 regulates MYB28/29 expression. Although IAA5, 6, and 19 are required for expression of $M Y B 28$, they are unlikely to directly regulate $M Y B 28$ because they are transcriptional repressors ${ }^{1}$. To identify transcription factors that might be direct targets 
a

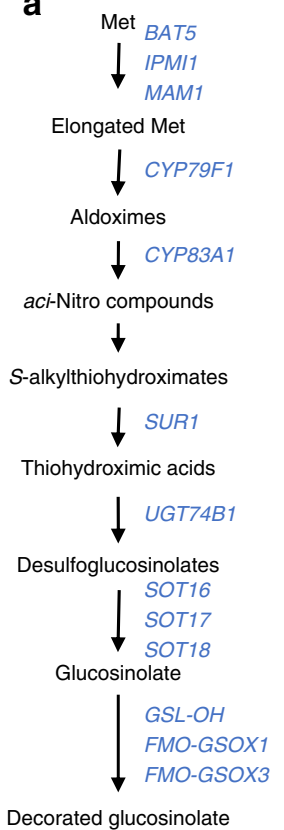

b

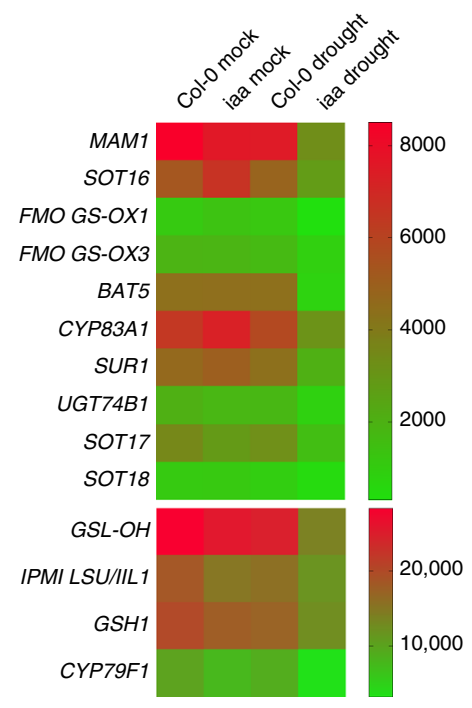

C

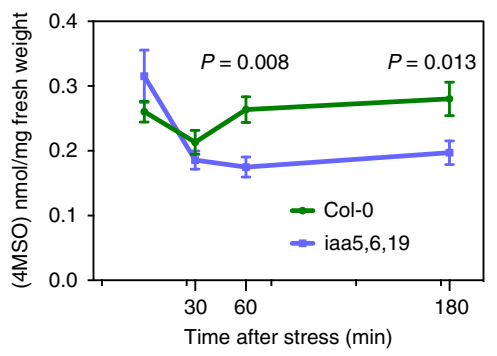

Fig. 1 The iaa5,6,19 mutant has lower aliphatic glucosinolate levels after dehydration stress. a Aliphatic glucosinolate biosynthetic pathway. The genes listed to the right are all down-regulated in the triple mutant compared to the wild type after dehydration. $\mathbf{b}$ Heat map showing that aliphatic glucosinolate biosynthetic genes are down regulated in the iaa5,6,19 mutant during desiccation stress. c Time course measurement of 4-MSO levels in 7-day-old seedlings of indicated genotypes at time intervals after application of dehydration stress. Results are presented as the means \pm SE of one experiment with 6 biological replicates consisting of $n=20$ pooled seedlings in each. Two independent experiments showed similar a trend. Statistical significance was determined by (Student's $t$-test). $P=0.008$ and 0.013 respectively for iaa5619 at 60 and 180 min of desiccation. Source data are provided as a Source Data file

of the Aux/IAAs, and that might regulate MYB28 expression, we searched our RNAseq data for factors that are up-regulated in the triple mutant in response to stress. One interesting candidate was WRKY63, also known as ABA OVERLY SENSITIVE3 (abo3) ${ }^{19}$. The abo3 mutant was originally isolated because it is hypersensitive to ABA in seedlings. Our qRT-PCR experiments confirmed that WRKY63 is up-regulated in response to dehydration in the triple mutant compared to the wild type (Fig. 3a). Examination of the promoter region of the WRKY63 gene revealed the presence of two tandemly repeated AuxRE elements ( -350 to -361 ), known to bind ARF transcription factors ${ }^{2}$. To determine if IAA19 binds to these sequences, we performed a ChIP-PCR analysis using a $r A A 19$-YPet-His-FLAG line. This line had previously been shown to rescue the iaa 19 mutant ${ }^{4}$. The results, shown in Fig. $3 \mathrm{~b}$, show that recovery of $A u x R E$ sequences is significantly enriched in the IP from rIAA19-YPet-His-FLAG compared to the control indicating that IAA19 binds to this sequence, presumably indirectly through an interaction with an ARF transcription factor.

To determine if WRKY63 might regulate MYB28/29 expression, we measured RNA levels in two 35 S:WRKY63 lines by qRTPCR and found that expression of both genes was reduced suggesting that WRKY63 acts to repress expression of the MYB genes (Fig. 3c) ${ }^{20}$. Finally, we measured drought tolerance of the $35 \mathrm{~S}$ :WRKY63 lines as well as the knockout mutant wrky63-1. As shown in Fig. $3 \mathrm{~d}$ and Supplementary Fig. 3b, the mutant has a normal response to water withholding. However, both overexpression lines are less drought tolerant consistent with reduced expression of $M Y B 28 / 29$. We note that WRKY63 is a member of small clade of 4 genes. It is possible that these genes have an overlapping function in regulation of $M Y B 28 / 29$. Our analysis of WRKY63 differs from the earlier work showing that the abo1 mutant is less drought tolerance ${ }^{19}$. The reason for this discrepancy is unclear.
GLS compounds regulate stomatal aperture. In considering how aliphatic GLSs may regulate drought response, we noted that these compounds have been reported to promote stomatal closure $^{21}$. In addition, the myrosinase TGG1 is one of the most abundant proteins in Arabidopsis guard cells. Experiments with the tgg1 tgg2 mutant that lacks canonical myrosinases indicate that these enzymes are required for the effect of GLS on stomata $^{21}$. Further, ITC, a GLS metabolite, closes stomata in Arabidopsis and Vicia faba $a^{2-24}$. Together these results indicate that myrosinase-dependent breakdown products of applied GLS promote stomatal closure. To determine if a defect in stomatal regulation might be responsible for reduced drought tolerance in the iaa5,6,19 and myb28 myb29 lines, we first examined the stomatal response to drought in epidermal peels. As expected, the stomata on Col-0 plants close in response to drought conditions (Fig. 4a). In contrast, the stomata on both mutant lines failed to respond. We next asked whether application of 4MSO would promote stomatal closure in wild-type and mutant plants. As a control we also applied abscisic acid (ABA) The results shown in Fig. $4 \mathrm{~b}$ demonstrate that all three genotypes respond to both $\mathrm{ABA}$ and 4-MSO. We further tested the effects of 4-MSO on light-induced opening of stomata from darkadapted plants. The results in Fig. 4c show that 4-MSO inhibits this response in Col-0 and iaa5,6,19 plants. These results suggest that the primary basis for reduced drought tolerance in the iaa 5, 6,19 line is failure to close stomata in drought conditions. To test this possibility, we applied 4-MSO to wild-type and mutant plants subjected to water withholding in pots. The results in Fig. 2a, b show that application of this GLS restores drought tolerance in the mutant to wild-type levels. To confirm that myrosinase is required for drought tolerance, we examined the response of the $\operatorname{tgg} 1 \operatorname{tgg} 2$ double mutant to PEG. The results show that the mutant line is less tolerant than wild type 

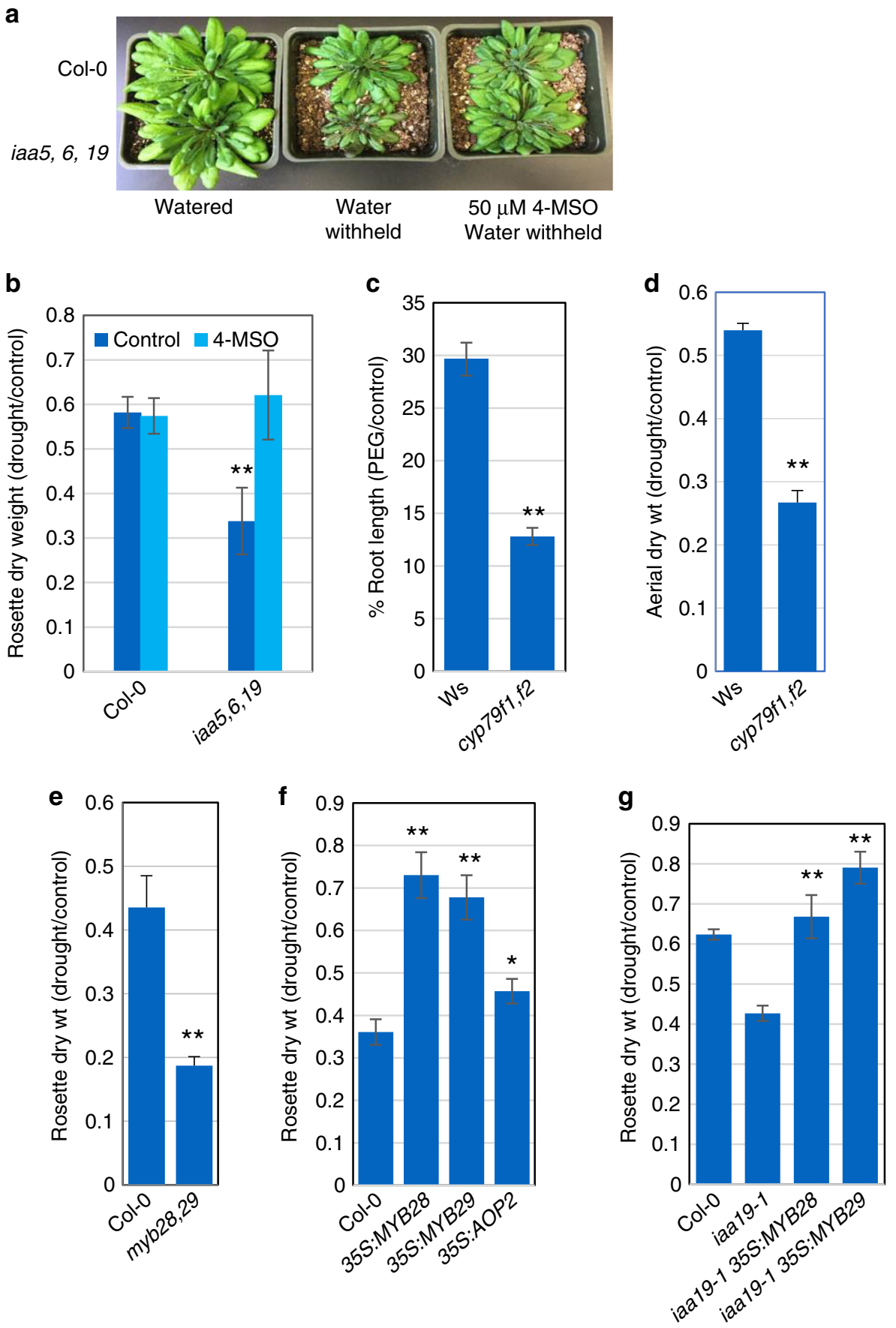

Fig. 2 Aliphatic glucosinolate deficiency is linked to decreased drought tolerance. a The iaa5,6,19 triple mutant is less drought tolerant than the wild type in a water withholding experiment. b Quantification of rosette dry weight from a. c cyp79f1,f2 double mutants are sensitive to growth on medium containing PEG compared to the wild-type Ws line. Results are presented as the mean \pm SE of one experiments with $n=12-15$ independent seedlings. $\mathbf{d}$ The cyp79f1,f2 line is less drought tolerant than Ws upon water withholding. e The myb28,29 double mutant is less drought tolerant compared to the wild type. $\mathbf{f} 35 \mathrm{~S}$ : MYB28, 35 S:MYB29 and 35 S:AOP2 plants display increase drought tolerance. $\mathbf{g}$ Overexpression of MYB28 or MYB29 restores drought tolerance to iaa19-1 plants. Results are presented as the means \pm SE of one experiment with $n=10$ independent pots. Each experiment was repeated at least twice. For $\mathbf{b}$-g, differences between mutants and Col-0 or Ws are significant at $p<0.05(*)$ and $p<0.01(* *)$ by two-tailed Student's $t$ test. Source data are provided as a Source Data file

confirming that it is a GLS metabolite that contributes to PEG tolerance (Supplementary Fig. 3c).

To determine if all GLS compounds promote stomatal closure, we applied indol-3-ylmethylglucosinolate (I3M), an indolic GLS that is abundant in Arabidopsis, to Col-0. The results in Fig. 4c demonstrate that $\mathrm{I} 3 \mathrm{M}$ is unable to inhibit light-induced stomatal opening in either Col-0 or the triple mutant, indicating that not all glucosinolates have the same efficacy. Similar results were obtained in an experiment where we tested the ability of I3M to promote stomatal closure in light grown plants (Fig. 4d). We also tested a second aliphatic GLS, sinigrin hydrate $(\mathrm{SH})$, that is abundant in many Arabidopsis ecotypes, although not Col- $0^{25}$. Like 4-MSO, SH promoted closure in Col-0 and in the abil-2 mutant (Fig. 4e). Thus, it is possible that this activity is restricted 
a

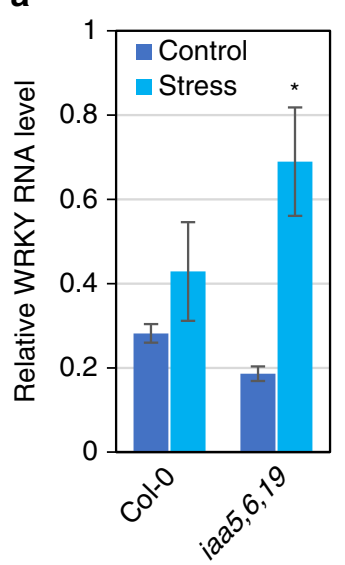

b
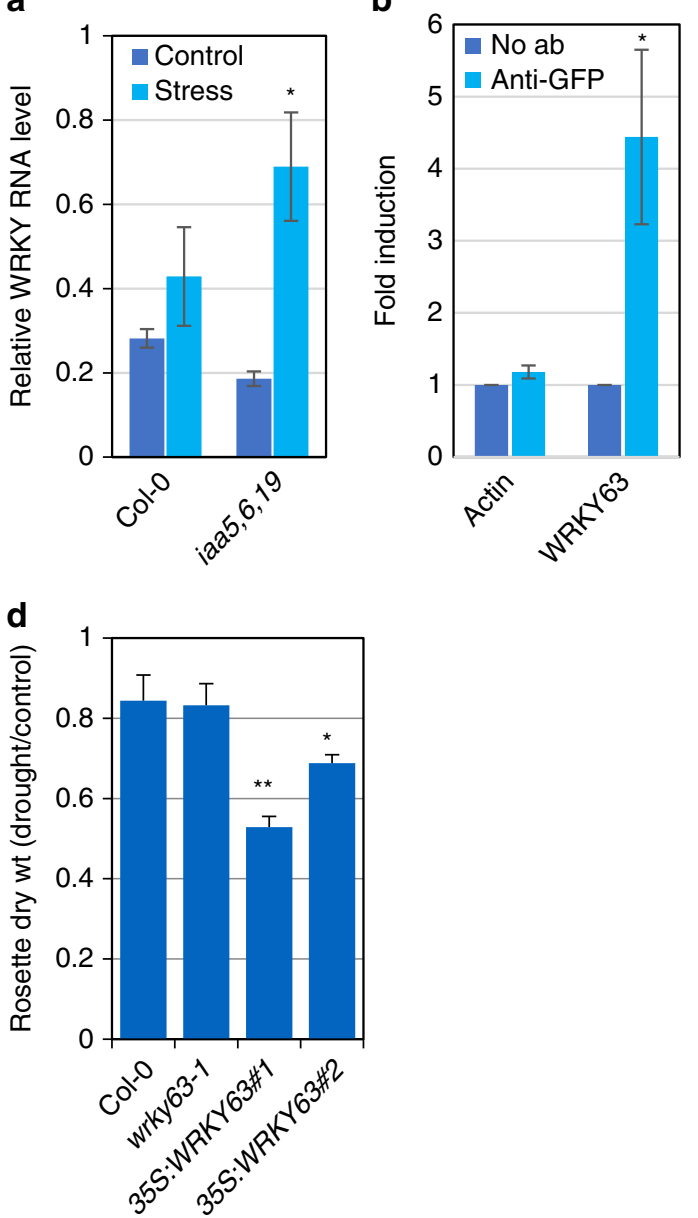

C

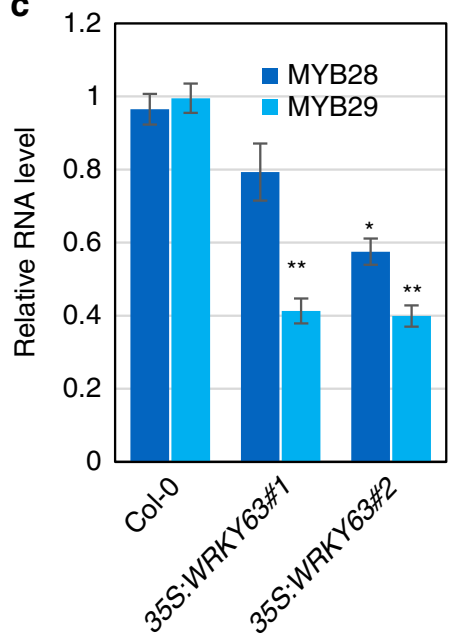

Fig. 3 Drought activates a transcriptional cascade to regulate GLS biosynthetic genes. a Relative WRKY63 RNA level in the iaa5,6,19 mutants compared to wild-type seedlings before and after desiccation for one hour. b IAA19 binds to a tandem repeat of AuxRE elements in the promoter of WRKY63 ( -350 to -361). ChIP was from the rAA19-YPet-His-FLAG line. Results are presented as the means \pm SE of 3 independent biological experiments with 3 technical replicates in each. Statistical significance was determined by two-tailed Student's t test. ${ }^{\star \star} P<0.05$. c Relative MYB28 and MYB29 RNA level in the seedlings of 35 S:WRKY63 lines. d 35 S:WRKY63 lines are less tolerant to drought than wild-type plants after water withholding in pots. Results are presented as the means \pm SE of one independent experiments with $n=10$ independent pots/plants. Differences are significant at $p<0.05(*)$ and $p<0.01(* *)$ by two-tailed Student's $t$ test. Source data are provided as a Source Data file

to aliphatic GLS compounds, although additional studies are required to explore this possibility further.

GLS action requires the receptor kinase GHR1. ABA is known to play a key role in stomatal regulation ${ }^{26}$. We investigated potential interactions between GLS and ABA by testing the response of iaa5,6,19 to ABA treatment. The results in Fig. $4 \mathrm{~b}$ show that the mutant line does respond to ABA, indicating that GLS is not required for the ABA response. We also used the abi12 mutant to address the role of ABA in the GSL response. ABI1 is a protein phosphatase $2 \mathrm{C}$ that functions in $\mathrm{ABA}$ signaling. The dominant abi1-1 mutant, which is deficient in ABA regulation of stomatal closure, has a normal response to GLS (Fig. 4e) ${ }^{26}$. These results suggest that GLS and ABA can act independently to regulate stomata. This is consistent with a recent study showing that GLS and ABA have an additive effect on stomatal closure ${ }^{27}$.

Reactive oxygen species (ROS) play an important role in stomatal regulation. One of the effects of $\mathrm{ABA}$ is to stimulate production of extracellular ROS through the RESPIRATORY BURST OXIDASE HOMOLOG D (RBOHD) ${ }^{26}$. Extracellular ROS than acts through the receptor kinase GUARD CELL HYDROGEN PEROXIDE-RESISTANT 1 (GHR1) to regulate the
SLOW ANION ACTIVATING CHANNEL $1^{26}$. Since ITCs, products of GLS metabolism, are known to promote stomatal closure via ROS, we wondered if GHR1 is required for response to $\mathrm{GLS}^{23}$. The results in Fig. 4f show that the grh 1 mutant is resistant to the effects of 4-MSO on stomata indicating that GLS likely acts through production of ROS.

Loss of TIR1/AFB proteins increases drought tolerance. Since loss of the auxin-sensitive repressors IAA5, IAA6, and IAA19 in the iaa5,6,19 results in decreased expression of GLS biosynthetic genes, we predict that the reduction in the levels of these proteins after auxin treatment would have the same effect. Indeed, treatment of seedlings with $10 \mu \mathrm{M}$ IAA for $2 \mathrm{~h}$ results in reduced expression of the GLS genes (Supplementary Fig. 3d). Further, we predict that mutations which stabilize the Aux/IAA proteins will increase both GLS levels and drought tolerance. The TIR1/AFB family of auxin co-receptors consists of 6 members in Arabidopsis that act in an overlapping fashion to regulate auxindependent transcription throughout the plant. To determine the role of the TIR1/AFBs in GLS regulation we measured GLS levels in two higher order tirl/afb lines, $a f b 1,3,4,5$ and $a f b 2,3,4,5^{28}$. Both lines had significantly higher GLS levels than either Col-0 or 
a

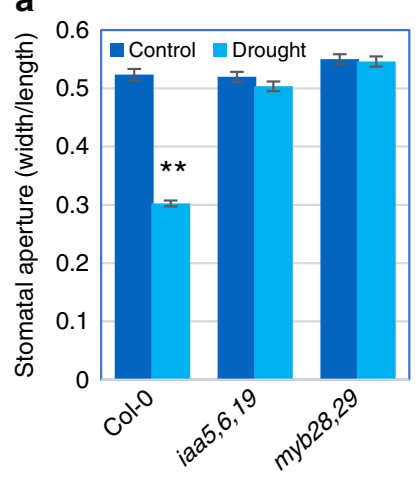

d

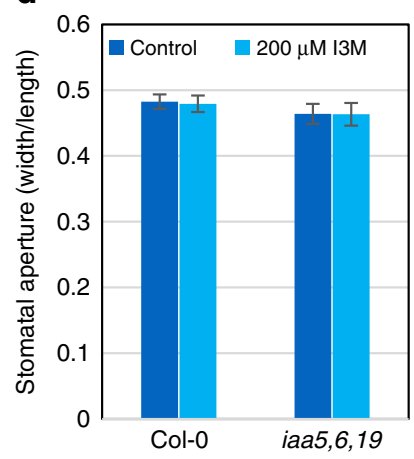

g

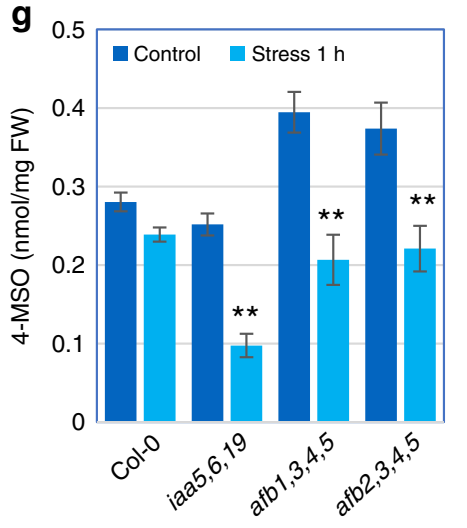

b

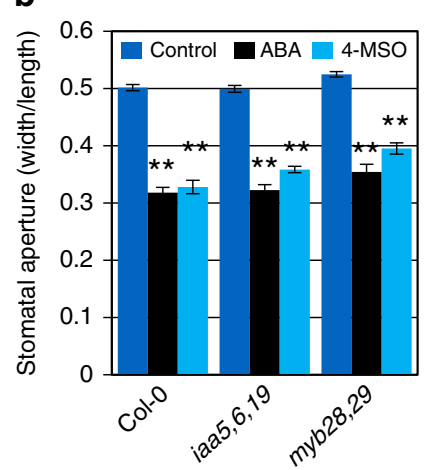

e

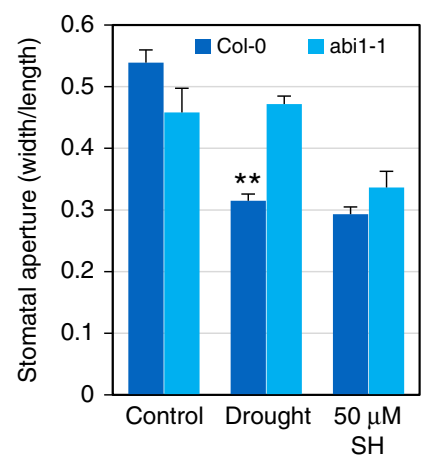

h

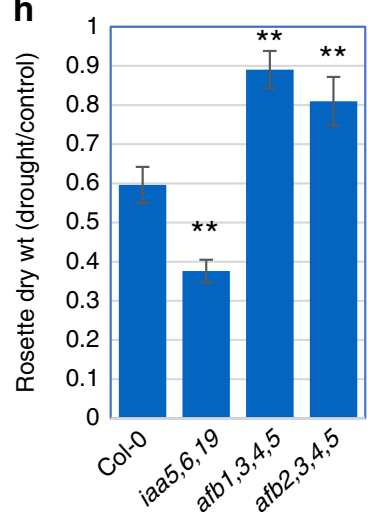

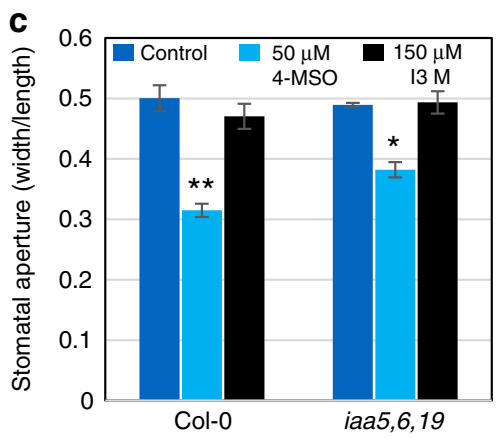

f

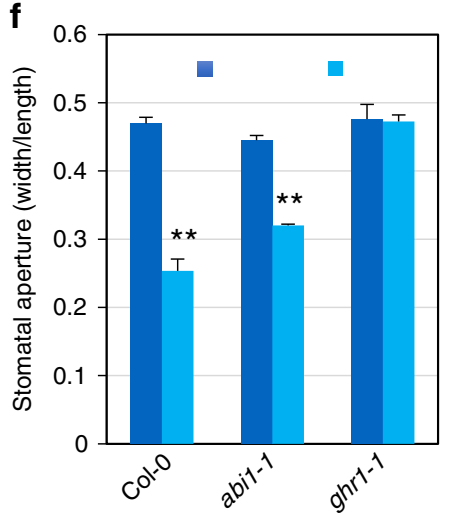

Fig. 4 Defects in stomatal regulation are responsible for decreased drought tolerance in glucosinolate deficient mutants. a Stomatal response to drought in Col-0, iaa5,6,19 and mybn28,29 lines. b Stomatal response to application of ABA and 4-MSO in Col-0, iaa5,6,19 and myb28,29. c 4-MSO inhibits lightinduced opening of stomata from dark-adapted plants while the indolic GLS I3M is ineffective. d Stomatal response to I3M in light-grown plants. e Response of the abi1-2 mutant to the aliphatic GLS sinigrin hydrate. f Response of the ghr1 mutant to 4-MSO, with abil-2 as a control. Unlike Col-0 or aib1-2, ghr1 does not respond to $50 \mu \mathrm{M} \mathrm{SH}$. g 4-MSO levels in tirl/afb mutants after 60 min desiccation treatment. $\mathbf{h}$ The tirl/afb mutants display increased tolerance to water withholding in pots. For stomatal closure experiments, $n=250-300$ stomata from 6 different leaves from 3 different plants grown in the same chamber and growth condidtions. Results are presented as the means \pm SE. Differences are significant at $p<0.05(*)$ and $p<0.01(* *)$ by two-tailed Student's $t$ test. Source data are provided as a Source Data file

iaa5,6,19 in 7-day-old seedlings (Fig. 4g). After one hour of desiccation, GLS levels dropped in all three mutant genotypes. In the case of the iaa5,6,19 line, GLS levels were much lower than Col-0, as observed previously. In contrast, GLS levels in $a f b 1,3,4,5$ and $a f b 2,3,4,5$ were similar to Col-0. We also determined the effects of water withholding on these genotypes. The results in Fig. 4h and Supplementary Fig. 3e show that both afb lines are significantly more drought tolerant than Col-0.

\section{Discussion}

Stomatal regulation is a complex process that requires the integration of multiple environmental inputs and signaling molecules, most notably ABA. Here we show that GLS compounds act as a recently evolved signal that regulates stomatal aperture. In Fig. 5, we present a model that can explain our results. In Arabidopsis, auxin acts independently of ABA to regulate stomatal aperture through its effect on GLS levels. GLS degradation products, perhaps ITCs but possibly other compounds, stimulate the formation of ROS. This is consistent with our data showing that the GHR1 receptor kinase is required for the GLS response. However, other scenarios are possible, and several important questions remain unanswered. For example, the source of the GLS and the site of myrosinase action are unknown. Although guard cells are known to have high levels of myrosinase, we have shown that GHR1 is required for the GLS response and it is extracellular ROS 


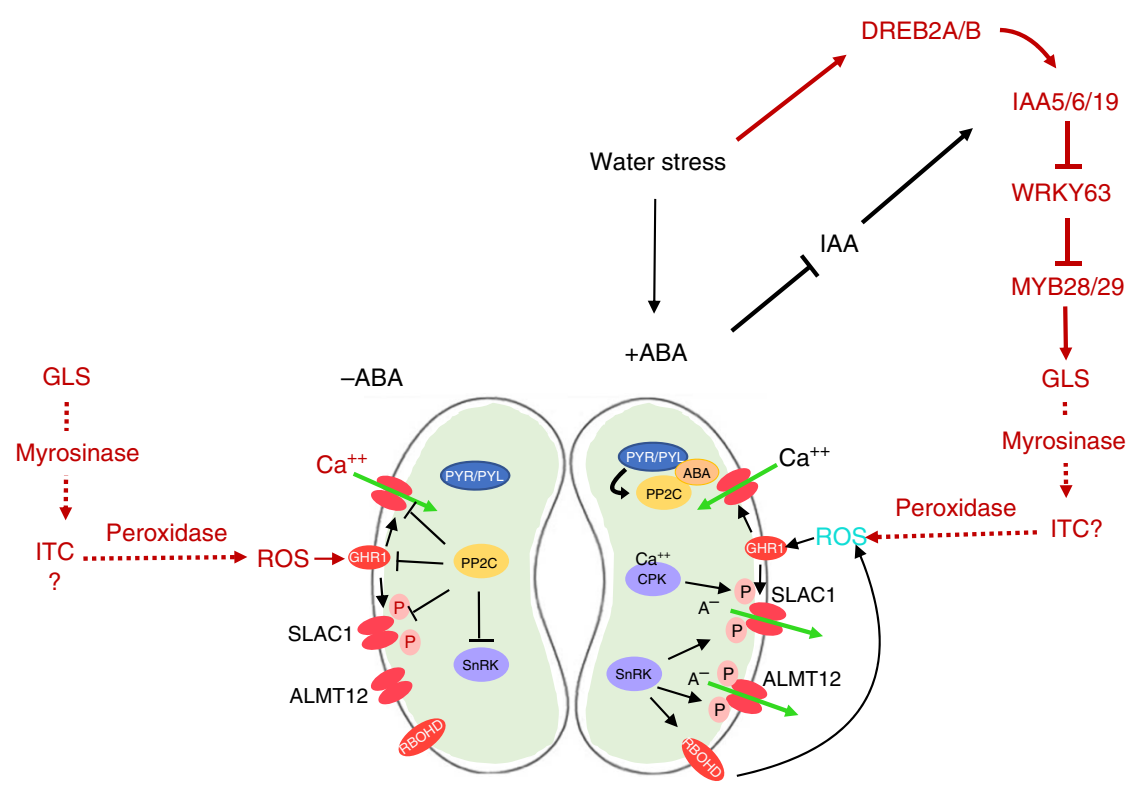

Fig. 5 A proposed model for GLS action during stomatal regulation. Adapted from ${ }^{26}$. GLS regulation diagramed in red. Water deficit induces DREB2A/B expression which promotes expression of IAA5/6/19 and maintenance of GLS levels. Myrosinase acts on GLS to produce degradation products, including isothiocyanates (ITC), that promote ROS production via a peroxidase ${ }^{22}$. An increase in ABA levels also results in decreased IAA levels in guard cells 27 which acts to stabilize IAA5/6/19. In the absence of water stress, GLS acts to promote stomatal closure via ROS production

that activates GHR1. Further, the precise identity of the active breakdown product is unknown. These and other important questions await further study.

Although GLS compounds are restricted to the Brassicaceae, allyl ITC has been shown to promote stomatal closure in Vicia faba via ROS, suggesting that some components of the GLS signaling pathway may be conserved ${ }^{24}$. Whether or not there is a source of endogenous ITC or related molecule in beans and other non-Brassicaceae remains to be determined. Regardless, our results suggest that other secondary metabolites, in addition to the classical plant hormones, have probably been recruited to perform signaling functions.

Why has auxin, a hormone typically associated with growth regulation, been recruited to regulate GLS biosynthesis? Many recent studies have described the connection between growth regulation and abiotic stress response. For example, gibberellic acid is known to play a key role in regulating plant growth in response to stress ${ }^{29}$. Although there is less information on the role of auxin in growth retardation during stress, we have shown that desiccation or osmotic treatment results in a rapid decrease in auxin response in seedlings, an effect that probably results in decreased growth ${ }^{4}$. By utilizing auxin to regulate stomatal aperture, the plant may integrate growth and stomatal regulation in drought conditions.

\section{Methods}

Plant materials and growth conditions. Arabidopsis thaliana seed were sterilized with chlorine fumes generated by mixing $49 \mathrm{ml}$ bleach and $1 \mathrm{ml}$ hydrochloric acid. Sterilized seeds were sown on $1 / 2$ strength MS media, stratified at $4^{\circ} \mathrm{C}$ for $2-3$ days, and grown in long day conditions $\left(16 \mathrm{~h}\right.$ light: $8 \mathrm{~h}$ dark) at $22^{\circ} \mathrm{C}$ with a light intensity of $\sim 110-130 \mu \mathrm{mol} \mathrm{m}^{-2} \mathrm{~s}^{-1}$. In addition to wild type Col- 0 and Ws lines, the following mutant lines were used as described: iaa19-1 and iaa5 iaa6 iaa19, dreb2a dreb2b (ref. ${ }^{4}$ ); cyp79f1 cyp79f2 (ref. $\left.{ }^{4}\right)$; myb28 myb29, 35 S:MYB28, 35 S: MYB29 (ref. ${ }^{17} \mathrm{~S}:$ WRKY63 OE (ref. ${ }^{20}$ ). abi1-2 (ref. ${ }^{30}$ ); ghr1 (ref. ${ }^{26}$ ).

Plasmid construction. rIAA19-YPet-His-Flag was constructed by recombineering a 1xYPet-6xHis-3xFlag tag to the C-terminus of the IAA19 gene in the Arabidopsis TAC clone JAtY59D10 (refs. ${ }^{4,31}$ ).

Transgenic plants. After floral dip of wild-type Col-0 plants ${ }^{32}$, single-insertional transgenic lines of rIAA19-YPet-His-Flag were selected by chi-square test from T2 plants on 1x Linsmaier \& Skoog (Caisson Labs, UT) plates containing $15 \mathrm{mg} / \mathrm{ml}$ glufosinate ammonium. The expression of the tagged TFs was confirmed by Western blotting. Homozygous transgenic lines were selected from the subsequent generation for bulking seeds.

RNAseq. Col-0 and iaa5,6,19 seedlings were grown on $1 / 2$ MS for 7 days and desiccated for $1 \mathrm{~h}$ on parafilm at room temperature. Total RNA was extracted from three independent biological replicates of each genotype using RNeasy Plant Min Kits (Cat\#79254, Qiagen, CA) and genomic DNA contamination was removed by digestion with Turbo DNase (Cat\# AM1907, BioRad, CA) and/or RNase-Free DNase (Cat\#79254, Qiagen). RNA quantity was checked by Bioanalyzer for quality control. Library construction and sequencing were performed at the IGM genomics center at UCSD. About 40 million reads were obtained for each sample. Raw reads were processed at Bioinformatics core facility, UCSD School of Medicine using Array Studio software. Alignments were performed using OSA4 and differential expression determined using DESeq2 ${ }^{33}$. Gene ontology analysis was performed using Panther $34,35,36$.

ChIP qPCR. For ChIP qPCR Col-0, recIAA19: YPet-His-FLAG and gWRKY63: WRKY63: eYFP plants were grown on $1 / 2$ MS for 7 days in the dark. Approximately $1 \mathrm{~g}$ tissues were collected and crosslinked with Pierce ${ }^{\mathrm{m}} \mathrm{Methanol-free} \mathrm{16 \% \text {For- }}$ maldehyde (w/v) (Cat\#28906, Thermo Fischer Scientific, Carlsbad, CA) diluted to $1 \%$, for $20 \mathrm{~min}$ and quenched with $2 \mathrm{M}$ glycine for $5 \mathrm{~min}$. Samples were flash frozen and ground in liquid nitrogen. Chromatin was prepared using EpiQuik Plant ChIP Kit (Cat\# P-2014-48, NY, USA) as per manufacturer's instructions. Chromatin was sheared at $4{ }^{\circ} \mathrm{C}$ by 16 cycles of $30 \mathrm{~s}$ on and $90 \mathrm{~s}$ off, on high settings using Bioruptor Plus sonicator device (Cat\# B01020002, Diagenode, US). IP was done with $20 \mu \mathrm{g}$ $\left(10 \mu \mathrm{L}, 2 \mu \mathrm{g} / \mu \mathrm{L}\right.$ of anti-GFP (\#A11122, Thermo Fisher, Carlsbad) at $4{ }^{\circ} \mathrm{C}$ overnight. Purified DNA was used for qRT-PCR using SYBR green dye in a CFX96 Real-Time System (Bio-Rad, Hercules, CA).

qRT-PCR. Total RNA was extracted using RNA RNeasy Plant Mini Kits (Cat\#79254, Qiagen) and genomic DNA contamination was removed by digestion with Turbo DNase (Cat\# AM1907, BioRad, CA) and/or RNase-Free DNase (Cat\#79254, Quiagen). cDNA was synthesized using SuperScript III (Cat\# 18080085, Life Technologies/Thermo Fischer, Carlsbad) and analyzed by quantitative PCR using PowerUp ${ }^{\text {mx }}$ SYBR $^{\circledR}$ Green Master Mix (Cat\#A25742, Thermo Fischer Scientific, Carlsbad, CA) on a CFX96 Real-Time System (Biorad, Hercules, CA). RNA levels were normalized against PP2A gene. Fold induction was calculated by ddCt method ${ }^{37}$. RNA levels were quantified using the Thermo Scientific NanoDrop $2000^{38}$. All qRT-PCR experiments were repeated three times. The oligos used for PCR are listed in Supplementary Table 2.

Glucosinolate Profiling. Blind experiments were conducted with 3 different genotypes as described above. For each assay 2 independent experiments with 6 independent biological replicates grown under same condition were used. 
Typically, 20-30 mg (fresh weight) of pooled 7 day-post-germination seedlings ( $n$ = 15-20) were sampled, freeze dried and sent to UC Davis at room temperature for GLS quantification. In total $400 \mu \mathrm{l} 90 \%(\mathrm{v} / \mathrm{v})$ methanol was added to each sample tube and stored at $-20^{\circ} \mathrm{C}$ before extraction. The tissues were disrupted with two $2.3 \mathrm{~mm}$ metal ball bearings in a paint shaker at room temperature and incubated at room temperature for $1 \mathrm{~h}$. Tissues were pelleted by centrifugation for $15 \mathrm{~min}$ at $2500 \mathrm{~g}$ and the supernatant was used for anion exchange chromatography in 96well filter plates. After methanol and water washing steps, the columns were incubated with sulfatase solution overnight. Desulfo-GLS were eluted and analyzed by HPLC $^{39}$

Drought stress assays. Osmotic stress assays were performed as described in ref. ${ }^{4}$. Briefly $35 \mathrm{ml} 1 / 2 \mathrm{MS}$ media with $1 \%$ sucrose and $0.8 \%$ Bactoagar was autoclaved and solidified in $120 \mathrm{~mm}$ square plates and perfused overnight with $50 \mathrm{ml}$ $30 \%$ filter sterilized PEG-8000 solution. PEG was drained the next day. 5-6 daypost-germination seedlings were transferred to either $1 / 2-\mathrm{MS}$ or $1 / 2-\mathrm{MS}+\mathrm{PEG}$ plates and incubated at $22{ }^{\circ} \mathrm{C}$ in long days. Root length and plant fresh weight were measured after 8 to 10 days.

Drought stress assays in soil were performed as described ${ }^{40,41}$ with minor modifications. 7 to 10-day-old seedlings were planted in sectors (two plants per sector) of $8 \mathrm{~cm} \times 8 \mathrm{~cm} \times 10 \mathrm{~cm}(\mathrm{~L} \times \mathrm{W} \times \mathrm{H})$ plastic pots. Plants were grown in a short-day chamber $(8 \mathrm{~h} / 16 \mathrm{~h}$. light/dark $)$ at $21^{\circ} \mathrm{C}$ during day $18{ }^{\circ} \mathrm{C}$ during night with light intensity of $110-130 \mu \mathrm{mol} \mathrm{m}^{-2} \mathrm{~s}^{-1}$. Seedlings were fertilized regularly. After 18-20 days, pots were saturated with water, drained and weighed. Subsequently, water was withheld for 18-22 days to reduce weight up to $50 \%$ and each pot was re-watered to $75 \%$ level by injecting $40-50 \mathrm{ml}$ of water in the middle of the pot with a 22-gauge needle. Pots were then subjected to re-drought for $12-15$ days to reduce pot weight to $50-60 \%$. Fresh weight and dry-weights of each rosette were measured. For dry weight rosettes were dried overnight at $65^{\circ} \mathrm{C}$ in a hot air oven. Drought stress assays were repeated three times with similar results.

In the experiments to determine the effects of GLS on drought tolerance, plants were treated as above except that water withholding began after two weeks of watering. At the same time, plants were sprayed with $50 \mu \mathrm{M} 4-\mathrm{MSO}, 50 \mu \mathrm{M}$ sinigrin hydrate or water 6 times during the first over the next 10 to 12 days. The 4 MSO and sinigrin were dissolved in water.

Stomata aperture measurement. Stomatal aperture experiments were performed using fully expanded young leaves from wild-type and mutant plants grown for 3-4 weeks in a growth room with $8 \mathrm{~h}$ of light $/ 16 \mathrm{~h}$ of dark. To determine the effects of GLSs and drought on stomatal closure, control plants and plants that were subjected to water withdrawal for 3-4 days were placed in the light overnight to open the stomata. Leaves were then incubated in closing solution ( $5 \mathrm{mM}$ MES-KOH [pH 6.15], $20 \mathrm{mM} \mathrm{KCl}$, and $1 \mathrm{mM} \mathrm{CaCl}_{2}$ ) under light $(110-130 \mathrm{mmol} / \mathrm{m} 2 / \mathrm{s})$ for $2.5 \mathrm{~h}$ followed by a further $2.5 \mathrm{~h}$ in the presence of $50 \mu \mathrm{M}$ 4-MSO (Glucoraphanin, Cat\# 0009445, Cayman Chemicals), $200 \mu \mathrm{M} \mathrm{I3M}$ (Cat\# $2503 \mathrm{~S}$, Alkemist Labs), or $20 \mu \mathrm{M}$ ABA. I3M was dissolved in DMSO. Control solutions contained comparable amounts of ethanol (ABA) or DMSO (I3M). Abaxial leaf epidermal peels were fixed on glass slide with coverslips ${ }^{21,42}$ using Hollister Medical Adhesive Spray. Stomatal apertures were measured with a light microscope (Nikon, Japan). The epidermal peels were examined under a $\times 40$ objective using the microscope. After image acquisition, widths and lengths of stomatal apertures were measured using the ImageJ software $(\mathrm{NIH}$ Bethesda, US). Six independent biological replicates (40-50 stomata from one seedling per replicate) were used for one experiment and at least three independent plants were used.

To determine the effects of GLS on stomatal opening, leaves were incubated in opening solution (10 mM MES-KOH [pH 6.15], $10 \mathrm{mM} \mathrm{KCl}$, and $10 \mathrm{mM}$ iminodiacetic acid) in the dark for $2.5 \mathrm{~h}$ with their adaxial surface upward. Upon transfer to the light, 50uM 4-MSO, $50 \mu \mathrm{M}$ sinigrin hydrate (Cat\# S1647, SigmaAldrich) $200 \mu \mathrm{M}$ I3M or $20 \mu \mathrm{M} \mathrm{ABA}$ were added to the solution. Stomatal aperture was measured as above.

Statistical analysis. GraphPad Prism 5.0 (San Diego, CA) and Microsoft Excel 2008 were utilized for preparing graphs and statistical analysis. ANOVA utilized for GLS analysis. For others two tailed $t$-tests were employed.

\section{Data availability}

Plant lines will be available through the Arabidopsis Stock Center. RNAseq data have been deposited into NCBI as GEO\#PRJNA549546 (https://www.ncbi.nlm.nih.gov/ bioproject/PRJNA549456/). The source data underlying Figs. 2b-g, 3a-d, 4a-h, Supplementary Figs. $1 \mathrm{a}-\mathrm{f}, 2 \mathrm{~b}$, and $3 c$, $\mathrm{d}$ are available as a source data file. The authors confirm that any other data supporting findings of this manuscript are available from the corresponsing author upon request.

Received: 15 March 2019 Accepted: 16 August 2019 Published online: 06 September 2019

\section{References}

1. Salehin, M., Bagchi, R. \& Estelle, M. SCFTIR1/AFB-based auxin perception: mechanism and role in plant growth and development. Plant Cell 27, 9-19 (2015).

2. Weijers, D. \& Wagner, D. Transcriptional responses to the auxin hormone. Annu. Rev. Plant. Biol. 67, 539 (2016).

3. Dello Ioio, R. et al. A genetic framework for the control of cell division and differentiation in the root meristem. Science 322, 1380-1384 (2008).

4. Shani, E. et al. Plant stress tolerance requires auxin-sensitive Aux/IAA transcriptional repressors. Curr. Biol. 27, 437-444 (2017).

5. Katz, E. et al. The glucosinolate breakdown product indole-3-carbinol acts as an auxin antagonist in roots of Arabidopsis thaliana. Plant J. 82, 547-555 (2015).

6. Malinovsky, F. G. et al. An evolutionarily young defense metabolite influences the root growth of plants via the ancient TOR signaling pathway. eLife $\mathbf{6}$, e29353 (2017)

7. Halkier, B. A. \& Gershenzon, J. Biology and biochemistry of glucosinolates. Annu. Rev. Plant. Biol. 57, 303-333 (2006).

8. Bednarek, P. et al. A glucosinolate metabolism pathway in living plant cells mediates broad-spectrum antifungal defense. Science 323, 101-106 (2009).

9. Clay, N. K., Adio, A. M., Denoux, C., Jander, G. \& Ausubel, F. M. Glucosinolate metabolites required for an Arabidopsis innate immune response. Science 323, 95-101 (2009).

10. Wittstock, U. \& Gershenzon, J. Constitutive plant toxins and their role in defense against herbivores and pathogens. Curr. Opin. Plant. Biol. 5, 300-307 (2002).

11. Fan, J. et al. Pseudomonas sax genes overcome aliphatic isothiocyanatemediated non-host resistance in Arabidopsis. Science 331, 1185-1188 (2011).

12. Chhajed, S., Misra, B. B., Tello, N. \& Chen, S. Chemodiversity of the glucosinolate-myrosinase system at the single cell type resolution. Front. Plant Sci. 10, 618 (2019).

13. Gigolashvili, T., Engqvist, M., Yatusevich, R., Müller, C. \& Flügge, U. I. HAG2/ MYB76 and HAG3/MYB29 exert a specific and coordinated control on the regulation of aliphatic glucosinolate biosynthesis in Arabidopsis thaliana. New Phytol. 177, 627-642 (2008)

14. Gigolashvili, T., Yatusevich, R., Berger, B., Müller, C. \& Flügge, U. I. The R2R3-MYB transcription factor HAG1/MYB28 is a regulator of methioninederived glucosinolate biosynthesis in Arabidopsis thaliana. Plant J. 51, 247-261 (2007).

15. Sonderby, I. E., Burow, M., Rowe, H. C., Kliebenstein, D. J. \& Halkier, B. A. A complex interplay of three R2R3 MYB transcription factors determines the profile of aliphatic glucosinolates in Arabidopsis. Plant Physiol. 153, 348-363 (2010).

16. Hirai, M. Y. et al. Omics-based identification of Arabidopsis Myb transcription factors regulating aliphatic glucosinolate biosynthesis. Proc. Nat Acad. Sci. USA 104, 6478-6483 (2007).

17. Sønderby, I. E. et al. A systems biology approach identifies a R2R3 MYB gene subfamily with distinct and overlapping functions in regulation of aliphatic glucosinolates. PLoS ONE 2, e1322 (2007).

18. Wentzell, A. M. et al. Linking metabolic QTLs with network and cis-eQTLs controlling biosynthetic pathways. PLoS. Genet. 3, 1687-1701 (2007).

19. Ren, X. et al. ABO3, a WRKY transcription factor, mediates plant responses to abscisic acid and drought tolerance in Arabidopsis. Plant J. 63, 417-429 (2010).

20. Van Aken, O., Zhang, B., Law, S., Narsai, R. \& Whelan, J. AtWRKY40 and AtWRKY63 modulate the expression of stress-responsive nuclear genes encoding mitochondrial and chloroplast proteins. Plant Physiol. 162, 254-271 (2013).

21. Zhao, Z., Zhang, W., Stanley, B. A. \& Assmann, S. M. Functional proteomics of Arabidopsis thaliana guard cells uncovers new stomatal signaling pathways. Plant Cell 20, 3210-3226 (2008).

22. Hossain, M. S. et al. Glucosinolate degradation products, isothiocyanates, nitriles, and thiocyanates, induce stomatal closure accompanied by peroxidase-mediated reactive oxygen species production in Arabidopsis thaliana. Biosci. Biotechnol. Biochem. 77, 977-983 (2013).

23. Khokon, M. A. et al. Allyl isothiocyanate (AITC) induces stomatal closure in Arabidopsis. Plant Cell Environ. 34, 1900-1906 (2011).

24. Sobahan, M. A. et al. Allyl isothiocyanate induces stomatal closure in Vicia faba. Biosci. Biotechnol. Biochem. 79, 1737-1742 (2015).

25. Francisco, M. et al. The defense metabolite, allyl glucosinolate, modulates Arabidopsis thaliana biomass dependent upon the endogenous glucosinolate pathway. Front. Plant Sci. 7, 774 (2016).

26. Munemasa, S. et al. Mechanisms of abscisic acid-mediated control of stomatal aperture. Curr. Opin. Plant. Biol. 28, 154-162 (2015).

27. Zhu, M. \& Assmann, S. M. Metabolic signatures in response to abscisic acid (ABA) treatment in Brassica napus guard cells revealed by metabolomics. Sci. Rep. 7, 12875 (2017). 
28. Prigge, M. J., Kadakia, N., Greenham, K. \& Estelle, M. Members of the Arabidopsis auxin receptor gene family are essential early in embryogeneis and have broadly overlapping functions. Preprint at https://www.biorxiv.org/ content/10.1101/529248v1

29. Colebrook, E. H., Thomas, S. G., Phillips, A. L. \& Hedden, P. The role of gibberellin signalling in plant responses to abiotic stress. J. Exp. Biol. 217, 67-75 (2014)

30. Saez, A. et al. Enhancement of abscisic acid sensitivity and reduction of water consumption in Arabidopsis by combined inactivation of the protein phosphatases type 2C ABI1 and HAB1. Plant Physiol. 141, 1389-1399 (2006).

31. Alonso, J. M. \& Stepanova, A. N. A recombineering-based gene tagging system for Arabidopsis. Methods Mol. Biol. 1227, 233-243 (2015).

32. Clough, S. J. \& Bent, A. F. Floral dip: a simplified method for Agrobacteriummediated transformation of Arabidopsis thaliana. Plant J. 16, 735-743 (1998).

33. Love, M. I., Huber, W. \& Anders, S. Moderated estimation of fold change and dispersion for RNA-seq data with DESeq2. Genome Biol. 15, 550 (2014).

34. Ashburner, M. et al. Gene ontology: tool for the unification of biology. The Gene Ontology Consortium. Nat. Genet. 25, 25-29 (2000).

35. $\mathrm{Mi}, \mathrm{H}$. et al. PANTHER version 11: expanded annotation data from Gene Ontology and Reactome pathways, and data analysis tool enhancements. Nucleic Acids Res. 45, D183-D189 (2017).

36. The Gene Ontology Consortium. Expansion of the Gene Ontology knowledgebase and resources. Nucleic Acids Res. 45, D331-D338 (2017).

37. Schmittgen, T. D. \& Livak, K. J. Analyzing real-time PCR data by the comparative C(T) method. Nat. Protoc. 3, 1101-1108 (2008).

38. Chapman, E. J. et al. Hypocotyl transcriptome reveals auxin regulation of growth-promoting genes through GA-dependent and -independent pathways. PLoS ONE 7, e36210 (2012).

39. Kliebenstein, D. J. et al. Genetic control of natural variation in Arabidopsis glucosinolate accumulation. Plant Physiol. 126, 811-825 (2001).

40. Bhaskara, G. B., Yang, T. H. \& Verslues, P. E. Dynamic proline metabolism: importance and regulation in water limited environments. Front. Plant Sci. 6 , 484 (2015).

41. Bates, L. S., Waldren, R. P. \& Teare, I. D. Rapid Determination of free proline for water-stress studies. Plant Soil 39, 205-207 (1973).

42. Fan, L. M. et al. Abscisic acid regulation of guard-cell $\mathrm{K}+$ and anion channels in Gbeta- and RGS-deficient Arabidopsis lines. Proc. Natl Acad. Sci. USA 105, 8476-8481 (2008).

\section{Acknowledgements}

We thank Jiyoung Park, Po-Kai Hsu, and Julian Schroeder for abil-21 and ghrl seeds and for help with stomatal aperture measurement, Eric Schmelz for assistance with freeze drying of samples, Jeongim Kim and Clint Chapple for cyp83al seeds, Venkatesan Sundaresan and Titima Tantikanjana for $c y p 79 f$ single and double mutant lines, and
Jim Whelan for wrky63-1 and 35 S::WRKY63 seeds. This work was supported by grants from the NIH (GM43644 to M.E.; J.R.E.), the Howard Hughes Medical Insitute (M.E. and J.R.E.), the NSF (IOS 1547796 and 1339125 to D.J.K.) the USDA National Institute of Food and Agriculture, (Hatch project number CA-D-PLS-7033-H to D.J.K.) and by the Danish National Research Foundation (DNRF99) grant.

\section{Author contributions}

M.S. and M.E. conceived of the project. M.S. prepared samples. M.T. analyzed RNAseq data. B.L. and E.K. performed glucosinolate assays. M.S. generated transgenic lines, performed genetic experiments, and performed physiological experiments. D.J.K. assisted with glucosinolate analysis. M.S. and M.E. wrote the manuscript. M.S., M.E., L.S., J.R.E., E.K. and D.J.K. edited the manuscript. J.R.E. and L.S. provided materials.

\section{Additional information}

Supplementary Information accompanies this paper at https://doi.org/10.1038/s41467019-12002-1.

Competing interests: The authors declare no competing interests.

Reprints and permission information is available online at http://npg.nature.com/ reprintsandpermissions/

Peer review information: Nature Communications thanks Hitoshi Kurumizaka and other anonymous reviewer(s) for their contribution to the peer review of this work. Peer reviewer reports are available

Publisher's note: Springer Nature remains neutral with regard to jurisdictional claims in published maps and institutional affiliations.

cc (i) Open Access This article is licensed under a Creative Commons Attribution 4.0 International License, which permits use, sharing, adaptation, distribution and reproduction in any medium or format, as long as you give appropriate credit to the original author(s) and the source, provide a link to the Creative Commons license, and indicate if changes were made. The images or other third party material in this article are included in the article's Creative Commons license, unless indicated otherwise in a credit line to the material. If material is not included in the article's Creative Commons license and your intended use is not permitted by statutory regulation or exceeds the permitted use, you will need to obtain permission directly from the copyright holder. To view a copy of this license, visit http://creativecommons.org/ licenses/by/4.0/.

(C) The Author(s) 2019 